\title{
A Phase 1, Open-Label Study in Healthy Subjects to Evaluate the Absolute Bioavailability of AG-221 by a Microtracer Approach
}

\author{
Xiaomin Wang · Jian Chen · Josephine Reyes - Simon Zhou • \\ Maria Palmisano · Yan Li (D)
}

To view enhanced content go to www.oncologytherapy-open.com Received: June 14, 2019 / Published online: July 19, 2019

(C) The Author(s) 2019

\section{ABSTRACT}

Introduction: The purpose of this study was to evaluate the absolute bioavailability (BA) of AG221 following a single oral dose of $100 \mathrm{mg} \mathrm{AG}$ 221 and an intravenous (IV) dose of $\sim 100 \mu \mathrm{g}$ AG-221 containing approximately $300 \mathrm{nCi}$ of $\left[{ }^{14} \mathrm{C}\right]-\mathrm{AG}-221$.

Methods: This was a phase 1, open-label study. Six subjects who met all of the inclusion criteria and none of the exclusion criteria were enrolled in the study. After an overnight fast of at least $10 \mathrm{~h}$, the subjects received an oral dose (coated tablet) of $100 \mathrm{mg}$ of AG-221 at $0 \mathrm{~h}$ on dosing day. Four hours after the oral dose, the subjects received $100 \mu \mathrm{g}$ AG-221 containing $\sim 300 \mathrm{nCi}$ of $\left[{ }^{14} \mathrm{C}\right]$-AG-221 administered as an IV bolus. Blood samples were collected and analyzed for plasma concentrations of AG-221 and $\left[{ }^{14} \mathrm{C}\right]-\mathrm{AG}-$ 221 using a validated liquid chromatography with tandem mass spectrometry (LC-MS/MS)

Enhanced Digital Features To view enhanced digital features for this article go to https://doi.org/10.6084/ m9.figshare. 8845160 .

X. Wang $\cdot$ J. Chen

Non-Clinical Development, Celgene Corporation, Summit, NJ, USA

J. Reyes · S. Zhou · M. Palmisano · Y. Li ( $₫)$

Translational Development and Clinical

Pharmacology, Celgene Corporation, Summit, NJ, USA

e-mail: yali@celgene.com system and high-performance liquid chromatography (HPLC) fractionation followed by accelerator mass spectrometry analysis (AMS), respectively. Safety was evaluated throughout the study.

Results: The absolute BA after a 100-mg oral dose of AG-221 was measured as $57.2 \%$. While the total clearance was $1.37 \mathrm{~L} / \mathrm{h}, \sim 1 / 60$ of the liver blood flow in a typical $70-\mathrm{kg}$ human subject, the first-pass extraction was estimated to be less than $2 \%$, assuming that the total clearance was entirely due to liver metabolism. Thus, the fraction of the AG-221 dose absorbed was at least 50\%. AG-221 was safe and well tolerated when given under fasted conditions in a single $100-\mathrm{mg}$ dose as a coated tablet with a microtracer $\left[{ }^{14} \mathrm{C}\right]-\mathrm{AG}-221$ solution, as few drug-related treatment-emergent adverse events (TEAEs) were reported. No clinically significant changes or findings were noted in the clinical laboratory evaluations, vital sign measurements, and electrocardiograms (ECGs) performed during this study.

Conclusions: In healthy subjects under fasting conditions, the absolute BA following oral administration of a 100-mg AG-221 tablet was $57.2 \%$. AG-221 was safe and well tolerated in healthy male subjects when administered as a single 100-mg film-coated tablet plus $100 \mu \mathrm{g}$ $\left[{ }^{14} \mathrm{C}\right]-\mathrm{AG}-221$ given intravenously.

Trial Registration: ClinicalTrials.gov identifier, NCT02443168.

Funding: Celgene Corporation. 
Keywords: Absolute bioavailability; Accelerator mass spectrometry; AG-221; Relapsed or refractory acute myeloid leukemia

\section{INTRODUCTION}

AG-221 (enasidenib, IDHIFA ${ }^{\circledR}$ ) is a novel, firstin-class, selective, oral, small-molecule inhibitor of the isocitrate dehydrogenase-2 (IDH2)-mutant protein, one of multiple genetic mutations that drive hematologic malignancies such as acute myeloid leukemia (AML) and solid tumors, making it a highly targeted therapeutic candidate for the treatment of both liquid and solid tumors [1-3]. The compound has been demonstrated to reverse histone and DNA hypermethylation and induce differentiation in IDH2-mutant-positive leukemia cell models as well as to reduce 2-hydroxyglutarate (2-HG, an oncometabolite synthesized by mutated IDH2 proteins) levels by $>90 \%$ in vitro and in vivo in subjects with cancers that harbor IDH2 mutations [1-3]. AG-221 is approved by the US Food and Drug Administration (FDA) for the treatment of adult patients with relapsed or refractory AML with an IDH2 mutation as detected by a FDA-approved test [4-6]. AG-221 efficacy was established from an open-label, single-arm, multicenter study, where it showed a combined response (i.e., complete response (CR) and complete response with partial hematologic recovery $(\mathrm{CRh}))$ rate and duration of $23 \%$ and 8.2 months, respectively, as well as a rate of conversion from transfusion dependence to transfusion independence of $34 \%$. The most common adverse reactions $(\geq 20 \%)$ included nausea, vomiting, diarrhea, elevated bilirubin, and decreased appetite [4-6].

The pharmacokinetics (PK) of AG-221 have been studied and characterized in both healthy volunteers and patients with hematologic malignancies [7-9]. PK data from subjects with advanced hematologic malignancies up to the 650-mg QD dose level demonstrated high plasma exposure, a long plasma half-life of AG221 after a single dose, high drug accumulation after multiple doses, and relatively high PK variability [4]. The mean time to $C_{\max }\left(T_{\max }\right)$ ranged from approximately 1 to $24 \mathrm{~h}$ [4]. There was an approximate $50 \%$ increase in $\mathrm{AUC}_{0-t}$ and $\mathrm{AUC}_{0-\text { inf }}$ and a $64 \%$ increase in $C_{\max }$ when AG221 was administered under fed conditions compared with fasted conditions, and the recommended starting dose of AG-221 is $100 \mathrm{mg}$ taken orally once daily with or without food [4]. Data from a human absorption, distribution, metabolism, and excretion (ADME) study showed that the overall mean recovery of total radioactivity (TRA) was approximately $82 \%$, with approximately $73.0 \%$ and $8.4 \%$ of the TRA recovered in feces and urine, respectively, during the 504-h postdose collection period [9]. These data indicate that TRA was primarily eliminated via fecal excretion. During the 240-h postdose period (the time frame during which metabolite profiling was performed), excreted metabolites and unchanged AG-221 accounted for greater than $47.1 \%$ and $24.9 \%$ of the administered dose, respectively. Of the $47.1 \%$ of the dose accounted for by excreted metabolites over the 240-h postdose period, fecal excretion of metabolites accounted for 39.9\% [9]. These results suggest that AG-221 is mainly cleared by metabolism, with the resulting metabolites eliminated primarily in the feces [9].

Assessing the BA of a formulation is a critical component of drug development because a common reason for failed drug development is poor drug BA [10]; hence, BA should be investigated as early as possible during drug development. Because AG-221 shows poor water solubility $(<0.0003 \mathrm{mg} / \mathrm{mL}$ in $\mathrm{pH} 7$ buffer, data on file) and is administered as an oral dose, it is important to assess the proportion of oral AG221 that reaches the systemic circulation in unchanged form. Knowing the absolute BA of the AG-221 tablet formulation will assist in understanding the overall disposition of the drug and interpreting other AG-221 PK data. In addition, this information will aid the development of new formulations for other indications, e.g., liquid or suspension formulations for pediatric tumor indications.

With that in mind, we performed a phase 1 , open-label study to evaluate the absolute BA of AG-221 in healthy adult male subjects. The primary objective was to evaluate the absolute BA of AG-221 following a single oral dose of $100 \mathrm{mg}$ AG-221 and an IV dose of $\sim 100 \mu \mathrm{g}$ AG- 
221 containing approximately $300 \mathrm{nCi}$ of $\left[{ }^{14} \mathrm{C}\right]-$ AG-221, and the secondary objective was to evaluate the tolerability and safety of AG-221 after a single 100-mg oral dose of a AG-221 tablet followed by an IV dose of $100 \mu \mathrm{g}$ AG-221 containing a microtracer of $\left[{ }^{14} \mathrm{C}\right]-\mathrm{AG}-221$ in healthy male subjects.

\section{METHODS}

\section{Ethics}

This study was conducted in accordance with the applicable US CFR governing the protection of human subjects, financial disclosure by clinical investigators, institutional review boards (IRBs), Investigational New Drug applications, applications for FDA approval to market a new drug, and radioactive drugs for certain research uses. Before the start of the study, the study protocol, informed consent form (ICF), and any other appropriate documents were submitted to the IRB (Salus Institutional Review Board, 2111 West Braker Lane, Suite 400, Austin, TX 78758, USA) with a cover letter or form listing the documents submitted, their dates of issue, and the site for which approval was sought. The investigator obtained ICFs from the subjects prior to any study-related procedures. During this study, there were no notable departures regarding compliance with applicable international good clinical practice (GCP) standards. The study was also in accordance with the 1964 Helsinki Declaration and its later amendments or comparable ethical standards.

\section{Study Design}

This was a single-center, open-label study conducted in six healthy adult males. Each subject participated in a screening phase (day -21 through -2$)$, a baseline visit, a treatment phase, and a follow-up phone call (7-10 days after the last assessment).

Prior to the start of the study, subjects underwent routine screening procedures and eligible subjects returned to the study center on day -1 for baseline assessments. Subjects who still qualified for participation were administered the study drug on the morning of day 1 and were domiciled at the clinical site from day 1 until day 3.

On day 1 , subjects received oral and IV doses of AG-221 in a fixed sequence: a 100-mg oral dose of AG-221 $(1 \times 100-\mathrm{mg}$ tablet $)$ was given after an overnight fast of $10 \mathrm{~h}$ and administered with $240 \mathrm{~mL}$ of room-temperature, noncarbonated water. Food and beverages (including water) were withheld from the subjects until at least $2 \mathrm{~h}$ following oral dosing. During the fasting period, water was allowed ad libitum except from $1 \mathrm{~h}$ prior to dosing until $2 \mathrm{~h}$ following dosing [excluding any water given with the investigational product (IP)]. Four hours after the oral dose, subjects were administered a $5-\mathrm{mL}$ IV solution containing $100 \mu \mathrm{g}$ of $\left[{ }^{14} \mathrm{C}\right]-\mathrm{AG}-221(\sim 300 \mathrm{nCi})$ in $0.25 \% \mathrm{DMSO} / 2.5 \%$ polysorbate $20 / 0.9 \%$ sodium chloride over approximately $2 \mathrm{~min}$. The IV solution was compounded from $\left[{ }^{14} \mathrm{C}\right]$-labeled AG-221 and unlabeled AG-221 powders.

Subjects were discharged from the unit upon completion of the 48-h PK blood draw on day 3 . Subjects returned to the unit for additional PK blood draws on days 5, 8, 11, 15, 18, 22, and 29.

The study design is presented in Fig. 1.

\section{Blood Collection for PK Analysis}

Blood samples $(\sim 3 \mathrm{~mL})$ were collected at the following time points for analysis of AG-221 concentrations: pre-dose (oral, $0 \mathrm{~h}$ ), 0.5, 1, 2, 3, 4 (before IV dose), 4.083 ( $5 \mathrm{~min}$ after IV dose), 4.25 (15 min after IV dose), 4.5, 5, 6, 7, 8, 10, 14, $18,24,48,96,168,240,336,408,504$, and $672 \mathrm{~h}$ (after the oral dose).

\section{Safety Assessment}

All subjects who received at least a single dose of AG-221 were included in the safety assessments, which included adverse event (AE) reporting, a review of concomitant medications/procedures, clinical laboratory safety tests, 12-lead ECG recordings, physical examinations (PEs), and vital sign measurements.

Subjects were monitored for safety throughout the study. All AEs and concomitant 

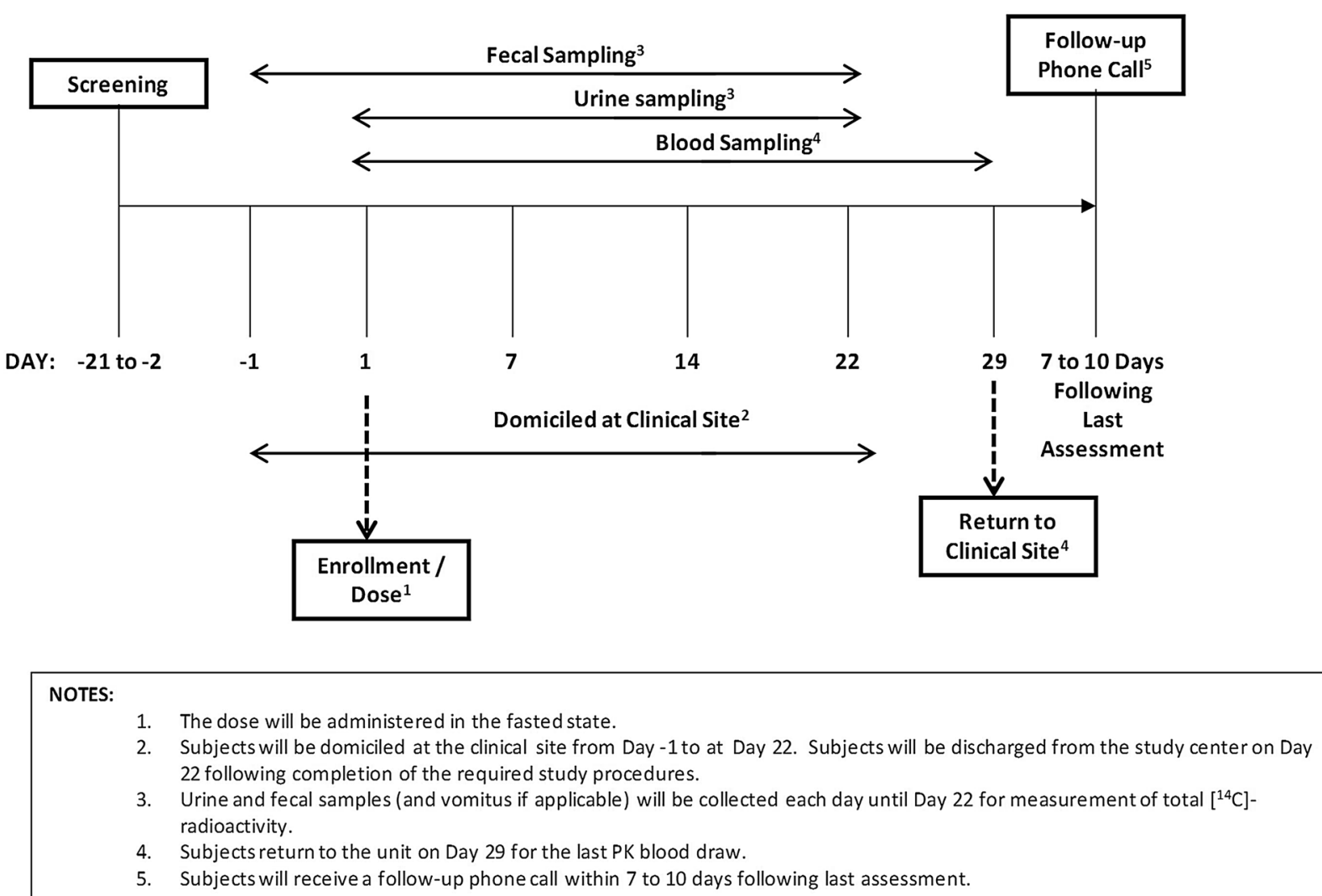

Fig. 1 Study design

medications were assessed and recorded throughout the study from the time the ICF was signed until study completion, and when made known to the investigator within 28 days after the last dose of IP [as well as serious adverse events (SAEs) that were made known to the investigator at any time thereafter and were suspected of being related to IP].

\section{Bioanalytical Methodology}

Concentrations of AG-221 from oral administration were analyzed using a validated method. The lower limit of quantification (LLOQ) for AG-221 in human plasma was $1.0 \mathrm{ng} / \mathrm{mL}$, with linearity demonstrated to $1000 \mathrm{ng} / \mathrm{mL}$ for AG221. The interassay coefficient of variance $(\% \mathrm{CV})$ values based upon the accepted calibration standards across the range were $\leq 6.4 \%$ for AG-221. Interassay precision values of AG-221, based upon the \%CV of the QC samples, were $\leq 6.7 \%$. Interassay accuracy values of AG-221, based upon the percent relative error (\%RE) of the QC samples, ranged from -2.5 to $7.0 \%$ for all QC levels. All plasma samples were analyzed by Covance (West Trenton, NJ, USA).

$\left[{ }^{14} \mathrm{C}\right]-\mathrm{AG}-221$ and AG-221 (Fig. 2) were from Nitto Denko Avecia, Inc. (8560 Reading Road, Cincinnati, OH 45215, USA) and Ash Stevens (18655 Krause Street, Riverview, MI 48193, USA), respectively. The specific radioactivity was $2.5 \mu \mathrm{Ci} / \mathrm{mg}$ and radiochemical purity by HPLC was greater than 99\%. The LLOQ for $\left[{ }^{14} \mathrm{C}\right]-\mathrm{AG}-221$ in human plasma was $0.00966 \mathrm{ng}$ $\mathrm{Eq} / \mathrm{mL}$, with linearity demonstrated to $5.00 \mathrm{ng}$ $\mathrm{Eq} / \mathrm{mL}$. The sample analysis was conducted at Accium BioSciences, Inc. (Seattle, WA, USA).

\section{PK Analyses}

The following plasma $\left[{ }^{14} \mathrm{C}\right]-\mathrm{AG}-221$ and nonlabeled AG-221 PK parameters were estimated by 
<smiles>CC(C)(O)CNc1nc(Nc2ccnc(C(F)(F)F)c2)nc(-c2ccc(C(F)(F)F)cn2)n1</smiles>

$\left[{ }^{1+} \mathrm{C}\right]-\mathrm{AG}-221$

Fig. 2 Structures of AG-221 and $\left[{ }^{14} \mathrm{C}\right]-\mathrm{AG}-221$

noncompartmental methods using actual elapsed time from dosing:

$\mathrm{AUC}_{0-t}$ : area under the plasma concentration-time curve (AUC) from time 0 to time $t$, where $t$ is the time of the last measurable concentration

$C_{\max }:$ maximum observed plasma concentration

$\mathrm{AUC}_{0-\text { inf }}$ : $\mathrm{AUC}$ from time 0 extrapolated to infinity

$T_{\max }$ : time to $C_{\max }$ obtained directly from the observed concentration versus time data

$t_{1 / 2}$ : terminal-phase elimination half-life

CL: total plasma clearance when dosed intravenously

$\mathrm{CL} / F$ : apparent total plasma clearance when dosed orally

$V_{\mathrm{z}} / F$ : apparent total volume of distribution when dosed orally

$V_{\text {ss }}$ : volume of distribution when dosed intravenously

$F_{\text {abs }}(\%)$ : absolute BA of AG-221

\section{Statistical Analyses}

The PK population included all subjects who received at least one dose of AG-221 and had at least one measurable datum. PK analyses were performed on data from the PK population. The safety population included all subjects who received at least one dose of AG-221. For subjects who withdrew or were discontinued from the study, all available PK and safety data<smiles>CC(C)(O)CNc1nc(Nc2ccnc(C(F)(F)F)c2)nc(-c2ccc(C(F)(F)F)cn2)n1</smiles>

AG-221

(including follow-up) were listed and summarized to the point of withdrawal/ discontinuation.

A total of six healthy adult male subjects were enrolled in the study. No formal sample size calculation was performed. The sample size was based on empirical rather than statistical considerations. Discontinued subjects could have been replaced at the discretion of the sponsor and investigator.

The plasma concentrations and PK parameters were summarized descriptively by treatment [mean, standard deviation, percent coefficient of variation (CV\%), geometric mean, geometric $\mathrm{CV} \%$, minimum, median, and maximum].

AEs were classified using the Medical Dictionary for Drug Regulatory Activities (version 17.1) classification system. All AEs were listed. A TEAE was defined as any AE that occurred after dosing of the first study drug or any $\mathrm{AE}$ that was already present pre-dose and worsened in either intensity or frequency following dosing. If the onset date/time of an $\mathrm{AE}$ was missing, the $\mathrm{AE}$ was defined as treatment emergent. TEAEs were summarized by frequency, severity, and relationship to study drug. The frequency of TEAEs (the number of TEAEs and the number of subjects experiencing a TEAE) were tabulated by system organ class and preferred term. In the by-subject analyses, a subject who suffered the same event more than once within the same level of summarization counted only once 
within the same treatment. AEs leading to death or to discontinuation from treatment and serious AEs were listed separately.

\section{RESULTS}

\section{Demographic and Other Baseline Characteristics}

Six subjects were enrolled in this study. All the subjects enrolled completed the study. A summary of the demographics is presented in Table 1. All enrolled subjects satisfied the inclusion and exclusion criteria, with no clinically significant abnormalities prior to first-dose administration, and the investigator approved all the subjects for study participation.

Of the subjects, $1(16.7 \%)$ and 5 (83.3\%) were Hispanic or Latino and not Hispanic or Latino, respectively. The majority of the subjects were white $(83.3 \%)$, with age ranging between 28 and 49 years, body weight ranging between 66.3 and $101.1 \mathrm{~kg}$, and body mass index ranging between 22.4 and $32.4 \mathrm{~kg} / \mathrm{m}^{2}$.

Table 1 Demographic and other baseline characteristics

\begin{tabular}{ll}
\hline Characteristic & Value $(\boldsymbol{N}=\mathbf{6})$ \\
\hline Mean age (range) in years & $39.5(28$ to 49$)$ \\
Mean height (range) in cm & $\begin{array}{l}176.47(168.1 \text { to } \\
186.6)\end{array}$ \\
Mean weight (range) in kg & 81.03 (66.3 to 101.1) \\
Mean BMI (range) in kg/m ${ }^{2}$ & $25.95(22.4$ to 32.4) \\
Race ( $n$ [\%]) & \\
White & $5(83.3)$ \\
Black or African American & - \\
American Indian or Alaska & $1(16.7)$ \\
Native & \\
Ethnicity ( $n$ [\%]) & $1(16.7)$ \\
Hispanic or Latino & $5(83.3)$ \\
Not Hispanic or Latino
\end{tabular}

$B M I$ body mass index, $N$ number of subjects, $n$ number of observations

\section{Quantification of $\left[{ }^{14} \mathrm{C}\right]-\mathrm{AG}-221$ by AMS}

A total of 120 plasma samples were analyzed to determine the concentrations of $\left[{ }^{14} \mathrm{C}\right]-\mathrm{AG}-221$ originating from an IV dose of $\left[{ }^{14} \mathrm{C}\right]-\mathrm{AG}-221$ using the validated HPLC-AMS method. The LLOQ for $\left[{ }^{14} \mathrm{C}\right]-\mathrm{AG}-221$ in human plasma was $0.00966 \mathrm{ng}-\mathrm{Eq} / \mathrm{mL}$, with linearity demonstrated to $5.00 \mathrm{ng}-\mathrm{Eq} / \mathrm{mL}$. Interassay precision values of $\left[{ }^{14} \mathrm{C}\right]-\mathrm{AG}-221$, based upon the $\% \mathrm{CV}$, were $\leq 7.72 \%$ (Table 2 ). Interassay accuracy values (\%RE) of $\left[{ }^{14} \mathrm{C}\right]-\mathrm{AG}-221$ ranged from 1.40 to $8.64 \%$ for all QC levels (Table 3), indicating a well-validated bioanalytical method to qualify $\left[{ }^{14} \mathrm{C}\right]-\mathrm{AG}-221$ from IV microtracer dosing.

\section{Absolute BA}

Figure 3 illustrates dose-normalized mean plasma concentration-time profiles of AG-221 after a single oral dose of $100 \mathrm{mg}$ and $100 \mu \mathrm{g}$ of an IV $\left[{ }^{14} \mathrm{C}\right]-A G-221$ dose containing $~ 300 \mathrm{nCi}$ of $\left[{ }^{14} \mathrm{C}\right]-A G-221$. Unlabeled AG-221 administered orally was quantified for $672 \mathrm{~h}$ post-dose using HPLC/MS/MS, whereas ${ }^{14} \mathrm{C}$-labeled AG221, administered via IV microdosing, was quantifiable for $408 \mathrm{~h}$ post-dose using HPLC/ AMS. After IV administration, the AG-221 PK profile showed two distinct phases: a rapid distribution phase followed by a slower elimination phase. However, after oral administration, the initial distribution phase was not apparent. The PK parameters for AG-221 after a single dose of $100 \mathrm{mg}$ AG-221 and an IV dose of $100 \mu \mathrm{g}$ $\left[{ }^{14} \mathrm{C}\right]-\mathrm{AG}-221$ containing $300 \mathrm{nCi}$ of $\left[{ }^{14} \mathrm{C}\right]-\mathrm{AG}-$ 221 are are summarized in Table 4.

As the amount administered following IV dosing was only $100 \mu \mathrm{g}$ containing $\sim 300 \mathrm{nCi}$ of $\left[{ }^{14} \mathrm{C}\right]-\mathrm{AG}-221$, the geometric mean peak plasma AG-221 concentration $C_{\max }(2.83 \mathrm{ng} /$ $\mathrm{mL}, 31.0 \%$ ) was lower following IV administration than the $C_{\max }$ following $100 \mathrm{mg}$ oral dosing $(703 \mathrm{ng} / \mathrm{mL}, \quad 16.4 \%)$. Similarly, the geometric mean systemic exposure of AG-221 as defined by $\mathrm{AUC}_{0-\text { inf }}$ was lower following IV infusion of AG-221 (73.0 $\mathrm{ng} \mathrm{h} / \mathrm{mL}, 21.9 \%$ ) than following oral AG-221 administration (41800 ng h/mL, 27.2\%). 
Table 2 Inter-run accuracy and precision based on $\left[{ }^{14} \mathrm{C}\right]-\mathrm{AG}-221$ standard curves

\begin{tabular}{|c|c|c|c|c|c|c|}
\hline Parameter & $\begin{array}{l}\text { Calibration \#1 } \\
(0.0649 \mathrm{dpm} / \\
\mathrm{mL})\end{array}$ & $\begin{array}{l}\text { Calibration \#2 } \\
(0.134 \mathrm{dpm} / \\
\mathrm{mL})\end{array}$ & $\begin{array}{l}\text { Calibration \#3 } \\
(1.23 \mathrm{dpm} / \\
\mathrm{mL})\end{array}$ & $\begin{array}{l}\text { Calibration \#4 } \\
(11.9 \mathrm{dpm} / \\
\mathrm{mL})\end{array}$ & $\begin{array}{l}\text { Calibration \#5 } \\
(29.0 \mathrm{dpm} / \\
\mathrm{mL})\end{array}$ & $\begin{array}{l}\text { Calibration \#6 } \\
(53.8 \mathrm{dpm} / \\
\mathrm{mL})\end{array}$ \\
\hline Mean & 0.0645 & 0.138 & 1.22 & 11.9 & 28.3 & 54.5 \\
\hline$\% \mathrm{CV}$ & 2.56 & 0.725 & 1.89 & 3.87 & 6.21 & 7.72 \\
\hline$\% \mathrm{RE}$ & -0.582 & 2.99 & 0.273 & 0.280 & -2.41 & 1.30 \\
\hline$n$ & 9 & 3 & 3 & 3 & 3 & 3 \\
\hline
\end{tabular}

Table 3 Inter-run accuracy and precision obtained with $\left[{ }^{14} \mathrm{C}\right]$-AG-221 quality-control samples

\begin{tabular}{lllll}
\hline $\begin{array}{l}\text { Performance } \\
\text { evaluation }\end{array}$ & $\begin{array}{l}\text { QC1 }(\mathbf{0 . 0 6 4 9} \mathrm{dpm} / \mathbf{m L}) \\
\text { LLOQ }\end{array}$ & $\begin{array}{l}\text { QC2 }(\mathbf{0 . 1 7 3} \mathrm{dpm} / \\
\mathbf{m L})\end{array}$ & $\begin{array}{l}\text { QC3 }(3.49 \mathrm{dpm} / \\
\mathbf{m L})\end{array}$ & $\begin{array}{l}\text { QC4 }(31.5 \mathrm{dpm} / \\
\mathbf{m L})\end{array}$ \\
\hline Mean & 0.0640 & 0.188 & 3.44 & 30.5 \\
Precision (\%CV) & 5.92 & 3.13 & 2.78 & 6.29 \\
Accuracy (\%RE) & 1.40 & 8.64 & 1.61 & 3.04 \\
$n$ & 15 & 15 & 15 & 15 \\
\hline
\end{tabular}

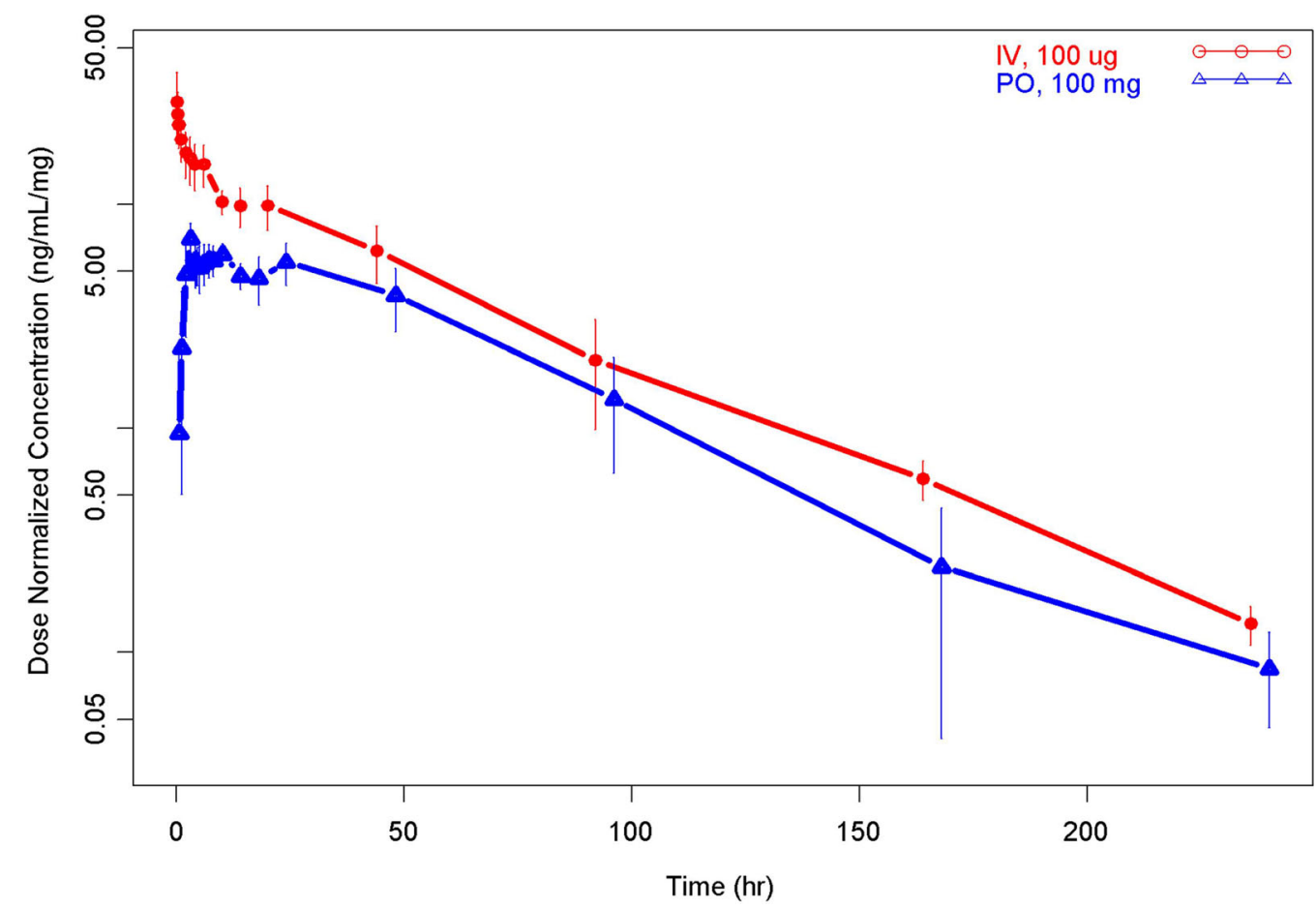

Fig. 3 Mean ( \pm standard deviation) dose-normalized AG-221 plasma concentration-time profiles: absolute BA assessment for oral and IV administration (red lines and symbols represent data from IV administration and blue lines and symbols represent data from oral administration) 
The geometric mean (geometric $\mathrm{CV} \%$ ) absolute BA $\left(F_{\text {abs }}\right)$ using the IV infusion treatment as the reference treatment was calculated as $57.2 \%( \pm 10.8 \%)$. The time to peak plasma AG-221 concentration ( $\left.T_{\max }\right)$ (median, range) was achieved at the end of infusion $(0.117 \mathrm{~h}$, range $=0.117-0.5 \mathrm{~h}$ ). Following oral administration, the drug was steadily absorbed and achieved $C_{\max }$ at $3.01 \mathrm{~h}$ (range $=3.0-24.0 \mathrm{~h}$ ). The plasma AG-221 terminal half-life $\left(t_{1 / 2}\right)$ after oral administration $(29.0 \mathrm{~h})$ was comparable to that following IV administration $(28.3 \mathrm{~h})$. The $\mathrm{CL} / F$ of AG-221 following oral administration was $2.39 \mathrm{~L} / \mathrm{h}$ vs. $1.37 \mathrm{~L} / \mathrm{h}$ following IV administration, which is approximately $1 / 60$ of the liver blood flow in a typical $70-\mathrm{kg}$ human subject, indicating a low first-pass extraction ratio for AG-221. The $V_{z} / F$ of AG-221 following oral administration was $100 \mathrm{~L}$ vs. $55.8 \mathrm{~L}$ for the $V_{\text {ss }}$ following IV administration, indicating that AG-221 is readily distributed out of the vasculature.

Of note, the intersubject variability of clearance expressed as the CV\% of the geometric mean was 27.2 and $21.9 \%$ for CL/F and CL from oral and IV administration, respectively, and the intersubject variability of absolute BA expressed as the $\mathrm{CV} \%$ of the geometric mean was $10.8 \%$, indicating the relatively high accuracy and precision of the AMS bioanalytical methodology. Consistent with this, individual dose-normalized $\mathrm{AUC}_{0 \text {-inf }}$ values for oral and IV administration were relatively tightly clustered, suggesting relatively low intersubject variability, as illustrated in Fig. 4.

\section{Safety}

Six subjects were given the study drug AG-221 as a single 100-mg coated tablet; 4 h later, all 6 subjects were also given an IV dose of $100 \mu \mathrm{g}$ of AG-221 containing approximately $300 \mathrm{nCi}$ of $\left[{ }^{14} \mathrm{C}\right]-\mathrm{AG}-221$. The 100-mg AG-221 film-coated tablet plus $100 \mu \mathrm{g}$ IV $\left[{ }^{14} \mathrm{C}\right]-\mathrm{AG}-221$ were well tolerated. Overall, 4 of 6 subjects $(66.7 \%)$ reported 7 TEAEs and 1 of 6 subjects (16.7\%) reported 1 TEAEs that were suspected of being related to the study drug. All TEAEs were mild in severity. There were no deaths reported, and no

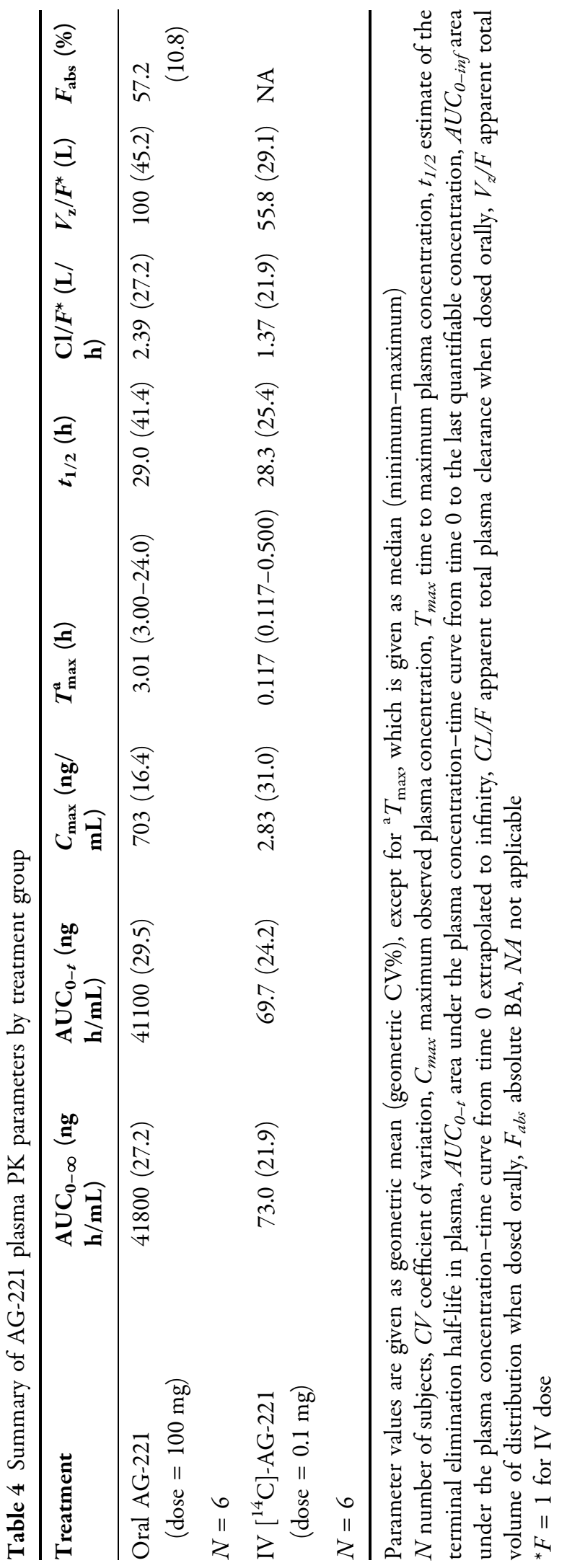




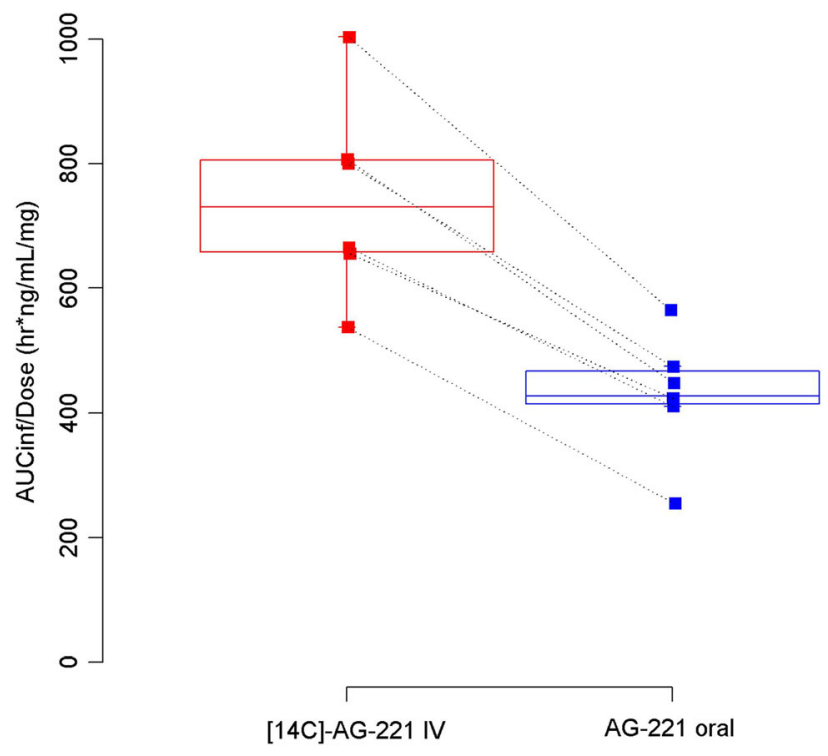

Fig. 4 Individual dose-normalized $\mathrm{AUC}_{0-\text { inf }}$ for $\mathrm{AG}-221$ (oral) and $\left[{ }^{14} \mathrm{C}\right]-\mathrm{AG}-221$ (IV). Squares represent individual values. Box plot provides median and 25\%/75\%

subjects experienced a SAE or discontinued due to TEAEs.

The most commonly reported TEAEs overall were general disorders and administration site conditions, with a total of 4 TEAEs reported by 3 subjects. There were 4 TEAEs related to conditions at the vessel puncture site and 1 TEAE of headache.

Sporadic out-of-range values occurred in multiple subjects on various clinical laboratory measurements. There were no clinically significant laboratory abnormalities, and no clinical laboratory values were reported as a TEAE. No marked changes in mean laboratory values over time or appreciable trends overall or within subjects were observed during the study.

\section{DISCUSSION}

Based on the guidance from the US FDA [11], from a PK perspective, the relative fraction of an extravascularly administered dose that is absorbed into the systemic blood circulation for a drug in a given formulation compared to that for a suspension, solution, or IV dose form can be estimated from relative/absolute BA studies. quartiles with whiskers to the last point within the $1.5 \times$ interquartile range. Squares connected by dotted lines are data from the same subject

Insightful PK information on distribution, elimination, the effects of formulation excipients on the absorption of the drug, dose linearity, and proportionality in the PK of active ingredients and (where appropriate) inactive ingredients can be obtained directly from data collected from BA studies. In addition, useful indirect information that is related to the properties of a drug substance before it enters the systemic blood circulation and plays an important role in the design and selection of formulations, such as the permeability, the rate of absorption, the in vivo dissolution profile, the in vivo disintegration profile for a given dosage form, and the influence of pre-systemic first-pass metabolizing enzymes and/or transports (such as P-glycoprotein, BCRP, etc.), can be further explored based on BA data. Therefore, the determination of the absolute BA in order to evaluate a drug's biopharmaceutic properties and to establish its pharmacology profile is recommended by various health authorities [12-14].

Traditionally, a crossover study design using either clinically relevant doses or doses that can provide sufficient plasma exposure to permit adequate characterization of the PK profiles is used to determine the absolute BA of a drug 
administered through a non-IV route versus an IV route [13]. Due to advances in mass spectrometry, the emergence of the concept of a microdosing/microtracer study has introduced an alternative approach for assessing the absolute BA [15-19]. In a microdosing/microtracer study, the subjects are administered a sub-pharmacologically active dose and blood/plasma samples are collected and analyzed for the parent drug or its metabolites. This approach first appeared in the late 1990s as a method of assessing human PK prior to full phase 1 clinical trials [15]. The sub-pharmacological dose administered in a human microdosing study normally leads to low drug plasma concentrations, so sensitive analytical technologies are needed to perform the requisite measurements over an appropriate time. For this reason, drugs are labeled with ${ }^{14} \mathrm{C}$, and AMS is used as it is a very sensitive analytical technique, capable of detecting drug concentrations in the femtogram to attogram $\left(10^{-15}\right.$ to $\left.10^{-18} \mathrm{~g}\right)$ range [15]. The advantages of the ${ }^{14} \mathrm{C}$-microtracer approach have been well described. First, as compared to the traditional crossover study approach, a stable IV formulation and associated toxicology packages are not required by regulatory agencies [15]; secondly, the potential intrasubject variability from crossover studies is eliminated [13]. In addition, the microtracer approach provides accurate plasma concentrations from IV and extravascular doses, and allows researchers (i.e., PK, pharmacodynamics, and DMPK scientists) to obtain more detailed information about the behavior of the drug in humans (i.e., exposure and elimination) $[10,13,20-22]$.

The primary objective of this phase 1 , openlabel study was to evaluate the absolute BA of AG-221 following a single oral dose of $100 \mathrm{mg}$ AG-221 and an IV dose of $\sim 100 \mu \mathrm{g}$ AG-221 containing approximately $300 \mathrm{nCi}$ of $\left[{ }^{14} \mathrm{C}\right]-\mathrm{AG}-$ 221 using the microtracer approach. The advantage of this approach is that accurate plasma concentrations from the IV dose (i.e., $0.1 \%$ of the oral dose of AG-221) can be determined by sensitive AMS and LC-MS/MS assays, thereby rendering the traditional crossover study design to determine the $F_{\text {abs }}$ of AG-221 as a tablet formulation unnecessary. In healthy subjects under fasting conditions, the geometric mean absolute BA following oral administration of a 100-mg AG-221 tablet was 57.2\%, indicating that AG-221 is well absorbed in humans. The results from this study suggest that AG-221 undergoes limited first-pass metabolism with low clearance. These results are consistent with data from a human mass balance ADME study, which showed that the recovery of total $\left[{ }^{14} \mathrm{C}\right]$ radioactivity over the 504 -h post dose period was $\sim 82 \%$ of the administered dose. The major route of AG-221 elimination was through the feces ( $\sim 73.0 \%$ of the radioactive dose), while urinary excretion was found to be only a minor elimination pathway $(\sim 8.4 \%$ of the radioactive dose). AG-221 was the most predominant component in the systemic blood circulating.

Data from the current absolute BA study provided helpful insights into the hepatic extraction of AG-221 (first-pass effect). The geometric mean systemic plasma clearance of AG-221 following the IV administration of a $100-\mu \mathrm{g}$ dose was $1.37 \mathrm{~L} / \mathrm{h}$ and the geometric mean plasma renal clearance of TRA in the ADME study was $0.0875 \mathrm{~L} / \mathrm{h}$. The partitioning of AG-221-related radioactivity in blood cells is limited, as reflected by a mean blood-to-plasma ratio of radioactivity ranging from approximately 0.38 to 0.81 from 0.5 to 672 -h postdosing. Therefore, assuming a well-stirred model and that AG-221 is eliminated through hepatic pathways, considering the typical hepatic blood flow of $87 \mathrm{~L} / \mathrm{h}$, the hepatic extraction ratio of AG-221 was small, suggesting a low firstpass effect for AG-221. Data from the current absolute BA study also provided helpful insights into the absorption profile of AG-221. The fraction of the AG-221 dose absorbed, assessed based on the absolute BA and the hepatic extraction of AG-221, was at least 50\%.

Finally, subjects who received both oral and IV administration of AG-221 showed good safety profiles. All TEAEs were of mild severity and resolved by the end of the study. There were no SAEs, and no subject withdrew from the study due to a TEAE. Drug-related TEAEs consisted of gastrointestinal disorders and headache. No clinically significant changes or findings were noted in clinical laboratory evaluations, vital sign measurements, or ECGs. 
A limitation of the current study is that the absolute BA of the AG-221 tablet was assessed in healthy volunteers. Although a study in healthy volunteers is likely to produce less PK variability than that in the intended patient population with potentially confounding factors such as underlying and/or concomitant disease and concomitant medications, pharmacological data collected from healthy subjects may not translate exactly to the intended patient population.

\section{CONCLUSIONS}

Based on the results from the microdosing study, the mean absolute BA after a 100-mg oral dose of AG-221 is $57.2 \%$. While the total clearance is $1.37 \mathrm{~L} / \mathrm{h}, \sim 1 / 60$ of the liver blood flow in a typical 70-kg human subject, the first-pass extraction is estimated to be less than $2 \%$, assuming the total clearance is by liver metabolism. Thus, the fraction of the AG-221 dose absorbed is at least $50 \%$. AG-221 was safe and well tolerated when given under fasted conditions as a single 100-mg dose, either as an oral solution or as a coated tablet with a microtracer $\left[{ }^{14} \mathrm{C}\right]-\mathrm{AG}-221$ solution, with few drug-related TEAEs reported. There were no clinically significant changes or findings noted in clinical laboratory evaluations, vital sign measurements, or ECGs during this study.

\section{ACKNOWLEDGEMENTS}

We thank the participants of the study and the site staff who made this study possible.

Funding. This study was funded by and the journal's rapid service fee was paid by Celgene Corporation. All authors had full access to all of the data in this study and take complete responsibility for the integrity of the data and accuracy of the data analysis.

Authorship. All named authors meet the International Committee of Medical Journal Editors (ICMJE) criteria for authorship for this manuscript, take responsibility for the integrity of the work as a whole, and have given final approval for the version to be published.

Disclosures. Xiaomin Wang is an employee of and holds equity ownership in Celgene Corporation. Jian Chen is an employee of and holds equity ownership in Celgene Corporation. Josephine Reyes is an employee of and holds equity ownership in Celgene Corporation. Simon Zhou is an employee of and holds equity ownership in Celgene Corporation. Maria Palmisano is an employee of and holds equity ownership in Celgene Corporation. Yan Li is an employee of and holds equity ownership in Celgene Corporation.

Compliance with Ethics Guidelines. This study was conducted in accordance with the applicable US CFR governing the protection of human subjects, financial disclosure by clinical investigators, institutional review boards (IRBs), Investigational New Drug applications, applications for FDA approval to market a new drug, and radioactive drugs for certain research uses.

All procedures performed in studies involving human participants were approved by the Salus Institutional Review Board, Austin, Texas, USA. They were also in accordance with the ethical standards of the institutional and/or national research committee and with the 1964 Helsinki Declaration and its later amendments or comparable ethical standards. ICFs were obtained from all individual participants included in the study.

Trial Registration. ClinicalTrials.gov identifier, NCT02443168.

Data Availability. The datasets obtained and/or analyzed during the current study are available from the corresponding author on reasonable request.

Open Access. This article is distributed under the terms of the Creative Commons Attribution-NonCommercial 4.0 International License (http://creativecommons.org/licenses/ by-nc/4.0/), which permits any noncommercial use, distribution, and reproduction in any medium, provided you give appropriate credit to the original author(s) and the source, provide 
a link to the Creative Commons license, and indicate if changes were made.

\section{REFERENCES}

1. Dogra R, Bhatia R, Shankar R, Bansal P, Rawal RK. Enasidenib: first mutant IDH2 inhibitor for the treatment of refractory and relapsed acute myeloid leukemia. Anticancer Agents Med Chem. 2018;18(14):1936-51.

2. Myers RA, Wirth S, Williams S, Kiel PJ. Enasidenib: an oral IDH2 inhibitor for the treatment of acute myeloid leukemia. J Adv Pract Oncol. 2018;9(4):435-40.

3. Pollyea DA, Tallman MS, de Botton S, Kantarjian HM, Collins R, Stein AS, et al. Enasidenib, an inhibitor of mutant IDH2 proteins, induces durable remissions in older patients with newly diagnosed acute myeloid leukemia. Leukemia. 2019. https:// doi.org/10.1038/s41375-019-0472-2

4. Celgene Corporation. Package insert for enasidenib. Summit, NJ: Celgene Corporation; 2017.

5. Click ZR, Seddon AN, Bae YR, Fisher JD, Ogunniyi A. New Food and Drug Administration-approved and emerging novel treatment options for acute myeloid leukemia. Pharmacotherapy. 2018;38(11):1143-54.

6. Talati C, Sweet K. Recently approved therapies in acute myeloid leukemia: a complex treatment landscape. Leuk Res. 2018;73:58-66.

7. Li Y, Connarn JN, Chen J, Tong Z, Palmisano M, Zhou S. Modeling and simulation of the endogenous CYP3A induction marker $4 \beta$-hydroxycholesterol during enasidenib treatment. Clin Pharmacol. 2019;11:39-50.

8. Li Y, Liu L, Gomez D, Chen J, Tong Z, Palmisano M, et al. Pharmacokinetics and safety of enasidenib following single oral doses in Japanese and Caucasian subjects. Pharmacol Res Perspect. 2018;6(6):e00436.

9. Tong Z, Atsriku C, Yerramilli U, Wang X, Li Y, Reyes $\mathrm{J}$, et al. Absorption, distribution, metabolism and excretion of an isocitrate dehydrogenase- 2 inhibitor enasidenib in rats and humans. Xenobiotica. 2019;49(2):200-10.

10. Shaik N, Hee B, Liang Y, LaBadie RR. Absolute oral bioavailability of glasdegib (PF-04449913), a smoothened inhibitor, in randomized healthy volunteers. Clin Pharmacol Drug Dev. 2019. https:// doi.org/10.1002/cpdd.692
11. US FDA. Bioavailability studies submitted in NDAs or INDs-general considerations. Silver Spring, MD: US FDA; 2019.

12. European Medicines Agency. Pharmacokinetic studies in man. Guideline. Amsterdam: European Medicines Agency; 1987.

13. Boulton DW, Kasichayanula S, Keung CF, Arnold ME, Christopher LJ, Xu XS, et al. Simultaneous oral therapeutic and intravenous ${ }^{14} \mathrm{C}$-microdoses to determine the absolute oral bioavailability of saxagliptin and dapagliflozin. Br J Clin Pharmacol. 2013;75(3):763-8.

14. Center for Drug Evaluation and Research. Waiver of in vivo bioavailability and bioequivalence studies for immediate-release solid oral dosage forms based on a biopharmaceutics classification system. Guidance for industry. Silver Spring, MD: US FDA; 2000.

15. Lappin G, Noveck R, Burt T. Microdosing and drug development: past, present and future. Expert Opin Drug Metab Toxicol. 2013;9(7):817-34.

16. Burt T, Yoshida K, Lappin G, Vuong L, John C, de Wildt SN, et al. Microdosing and other phase 0 clinical trials: facilitating translation in drug development. Clin Transl Sci. 2016;9(2):74-88.

17. Sudo K. Microdosing for reduction of the time and resources for drug development. Drug Metab Pharmacokinet. 2007;22(5):327.

18. Ings RM. Microdosing: a valuable tool for accelerating drug development and the role of bioanalytical methods in meeting the challenge. Bioanalysis. 2009;1(7):1293-305.

19. Burt T, Vuong LT, Baker E, Young GC, McCartt AD, Bergstrom M, et al. Phase 0, including microdosing approaches: applying the three Rs and increasing the efficiency of human drug development. Altern Lab Anim. 2018;46(6):335-46.

20. Vishwanathan K, So K, Thomas K, Bramley A, English S, Collier J. Absolute bioavailability of osimertinib in healthy adults. Clin Pharmacol Drug Dev. 2019;8(2):198-207.

21. Raje S, Callegari E, Sahasrabudhe V, Vaz A, Shi H, Fluhler E, et al. Novel application of the two-period microtracer approach to determine absolute oral bioavailability and fraction absorbed of ertugliflozin. Clin Transl Sci. 2018;11(4):405-11.

22. Cho YS, Lim HS, Han S, Yoon SK, Kim H, Cho YL, et al. Single-dose intravenous safety, tolerability, and pharmacokinetics and absolute bioavailability of LCB01-0371. Clin Ther. 2019;41(1):92-106. 